\title{
A PET/CT-Based Strategy Is a Stronger Predictor of Survival Than a Standard Imaging Strategy in Patients with Head and Neck Squamous Cell Carcinoma
}

\author{
Max Rohde ${ }^{1,2}$, Anne L. Nielsen ${ }^{3}$, Manan Pareek ${ }^{4}$, Jørgen Johansen ${ }^{5}$, Jens A. Sørensen ${ }^{6}$, Anabel Diaz ${ }^{7}$, Mie K. Nielsen ${ }^{7}$, \\ Janus M. Christiansen ${ }^{7}$, Jon T. Asmussen ${ }^{7}$, Nina Nguyen ${ }^{7}$, Oke Gerke ${ }^{2,8}$, Anders Thomassen ${ }^{2}$, Abass Alavi ${ }^{9}$, \\ Poul Flemming Høilund-Carlsen ${ }^{2,3}$, and Christian Godballe ${ }^{1,2}$ \\ ${ }^{1}$ Department of ORL-Head and Neck Surgery, Odense University Hospital, Odense, Denmark; ${ }^{2}$ Department of Clinical Research, \\ University of Southern Denmark, Odense, Denmark; ${ }^{3}$ Department of Nuclear Medicine, Odense University Hospital, Odense, \\ Denmark; ${ }^{4}$ Cardiology Section, Department of Internal Medicine, Holbk Hospital, Holbk, Denmark; ${ }^{5}$ Department of Oncology, \\ Odense University Hospital, Odense, Denmark; ${ }^{6}$ Department of Plastic Surgery, Odense University Hospital, Odense, Denmark; \\ ${ }^{7}$ Department of Radiology, Odense University Hospital, Odense, Denmark; ${ }^{8}$ Centre of Health Economics Research, University of \\ Southern Denmark, Odense, Denmark; and ${ }^{9}$ Department of Radiology, Hospital of the University of Pennsylvania, Philadelphia, \\ Pennsylvania
}

Our purpose was to examine whether staging of head and neck squamous cell carcinoma (HNSCC) by upfront ${ }^{18} \mathrm{~F}-\mathrm{FDG}$ PET/CT (i.e., on the day of biopsy and before the biopsy) discriminates survival better than the traditional imaging strategies based on chest $\mathrm{x}$-ray plus head and neck MRI (CXR/MRI) or chest CT plus head and neck MRI (CCT/MRI). Methods: We performed a masked prospective cohort study based on paired data. Consecutive patients with histologically verified primary HNSCC were recruited from Odense University Hospital from September 2013 to March 2016. All patients underwent CXR/MRI, CCT/ $\mathrm{MRI}$, and PET/CT on the same day. Tumors were categorized as localized (stages I and II), locally advanced (stages III and IVB), or metastatic (stage IVC). Discriminative ability for each imaging modality with respect to HNSCC staging were compared using Kaplan-Meier analysis, Cox proportional hazards regression with the Harrell C-index, and net reclassification improvement. Results: In total, 307 patients with histologically verified HNSCC were included. Use of PET/CT significantly altered the stratification of tumor stage when compared with either CXR/MRI or CCT/MRI $\left(\chi^{2}, P<0.001\right.$ for both). Cancer stages based on $\mathrm{PET} / \mathrm{CT}$, but not CXR/MRI or CCT/MRI, were associated with significant differences in mortality risk on Kaplan-Meier analyses ( $P \leq 0.002$ for all PET/CT-based comparisons). Furthermore, overall discriminative ability was significantly greater for $\mathrm{PET} /$ CT (C-index, 0.712) than for CXR/MRI (C-index, 0.675; $P=$ 0.04 ) or CCT/MRI (C-index, 0.657; $P=0.02$ ). Finally, PET/CT was significantly associated with a positive net reclassification improvement when compared with CXR/MRI $(0.184, P=0.03)$ but not CCT/MRI $(0.094 \%, P=0.31)$. Conclusion: Tumor stages determined by PET/CT were associated with more distinct prognostic properties in terms of survival than those determined by standard imaging strategies.

Received Jun. 9, 2017; revision accepted Aug. 24, 2017.

For correspondence or reprints contact: Max Rohde, Department of ORLHead and Neck Surgery, Odense University Hospital, Sdr. Boulevard 29, 5000 Odense C, Denmark.

E-mail: max.rohde@rsyd.dk

Published online Sep. 1, 2017.

COPYRIGHT (C 2018 by the Society of Nuclear Medicine and Molecular Imaging.
Key Words: computed tomography; head and neck squamous cell carcinoma; magnetic resonance imaging; positron emission tomography; staging; survival

J Nucl Med 2018; 59:575-581

DOI: 10.2967/jnumed.117.197350

$\mathbf{T}$ he incidence of head and neck squamous cell carcinoma (HNSCC) is rapidly increasing because of the aging of the population and increasing colonization with human papillomavirus $(1,2)$. Accurate assessment of tumor spread, that is, staging, is of critical importance for ensuring appropriate treatment and surveillance and for determining prognosis (3-6). Localized disease is most often treated with curative intent, whereas metastatic disease generally hampers definitive treatment and leads to a palliative treatment strategy (4). Proper diagnostic imaging is fundamental to ensure directed biopsies for histologic verification and precise assessment of disease extent (7).

In European head and neck cancer centers, a combination of chest x-ray plus head and neck MRI (CXR/MRI) has long been the standard clinical imaging strategy for evaluating patients with primary HNSCC $(8,9)$. The U.S. National Comprehensive Cancer Network guidelines recommend chest CT plus head and neck MRI (CCT/ MRI) for oral, pharyngeal, or laryngeal cancer and consider PET/ CT for clinical stage 3-4 (10). However, studies have suggested that PET/CT may be more accurate for TNM classification of HNSCC (11), particularly for detection of metastatic disease and synchronous cancers $(7,12-17)$. Still, the clinical implications of an upfront diagnostic PET/CT scan for HNSCC with respect to survival and prognosis are unclear. The objective of this study was thus to examine whether cancer stages determined by a PET/CT-based imaging strategy discriminate survival better than strategies based on either chest CXR/MRI or CCT/MRI in patients diagnosed with HNSCC.

\section{MATERIALS AND METHODS}

A masked prospective cohort study based on paired data was performed and reported according to the "Strengthening the Reporting of 
Observational Studies in Epidemiology" statement (18). The data were used for detailed analyses of staging and discriminative ability among the different imaging modalities. The study was conducted in accordance with good clinical practices and the Declaration of Helsinki. Permission was granted from the local ethics committee (project no. S_20120217) and the Danish Data Protection Agency (journal no. $12 / 26356$ ), and written informed consent was obtained from all included patients. The project was implemented without the involvement of private organizations or companies.

\section{Setting and Participants}

The study design, setting, and methods have been described previously (19). We considered consecutive patients with suspected primary oral, pharyngeal, or laryngeal carcinoma referred (in accordance with the Danish fast-track program (20)) to the Head and Neck Cancer Center, Odense University Hospital, from September 2013 to March 2016 (both inclusive). Clinical examination, including nasopharyngolaryngoscopy and neck ultrasound, was performed upon referral by an experienced head and neck cancer specialist. The patient was offered inclusion in the study if the suspected HNSCC was histologically confirmed (Fig. 1).

Exclusion criteria included allergy or intolerance to iodine contrast medium, use of high-dose systemic steroids (prednisone, $>50 \mathrm{mg} / \mathrm{d}$ ), impaired renal function (plasma creatinine $>90 \mu \mathrm{mol} / \mathrm{L}$ for women and $>105 \mu \mathrm{mol} / \mathrm{L}$ for men or previously diagnosed kidney disease), or inability to cooperate.

Patients underwent CXR/MRI, CCT/MRI, and PET/CT upfront (i.e., on the day of biopsy and before the biopsy) to improve the quality of staging and diagnostic interpretation. Acquiring imaging before biopsy is the standard procedure in the Danish fast-track program. The chest CT scan was derived from the PET/CT examinations to minimize radiation. PET/CT was performed with CT scans of full diagnostic quality. The MRI results used for both CXR/MRI and CCT/MRI were based on a single MRI examination.

\section{Imaging Techniques}

PET/CT data were acquired on a hybrid PET/CT scanner (Discovery 690, 710, VCT, or RX; GE Healthcare).

${ }^{18} \mathrm{~F}-\mathrm{FDG}(4 \mathrm{MBq} / \mathrm{kg}$ ) was injected intravenously after a fast of at least $4 \mathrm{~h}$. The PET scan was acquired using a standard whole-body protocol extending from the vertex to the thigh, at $2 \frac{1}{2}$ min per bed position. PET data were reconstructed into transaxial slices with a matrix size of $128 \times 128$ (pixel size, $5.47 \mathrm{~mm}$ ) or $256 \times 256$ (pixel size, $2.73 \mathrm{~mm}$ ) and a slice thickness of $3.27 \mathrm{~mm}$ using iterative 3-dimensional ordered-subsets expectation maximization. A multislice, diagnostic-quality CT scan with

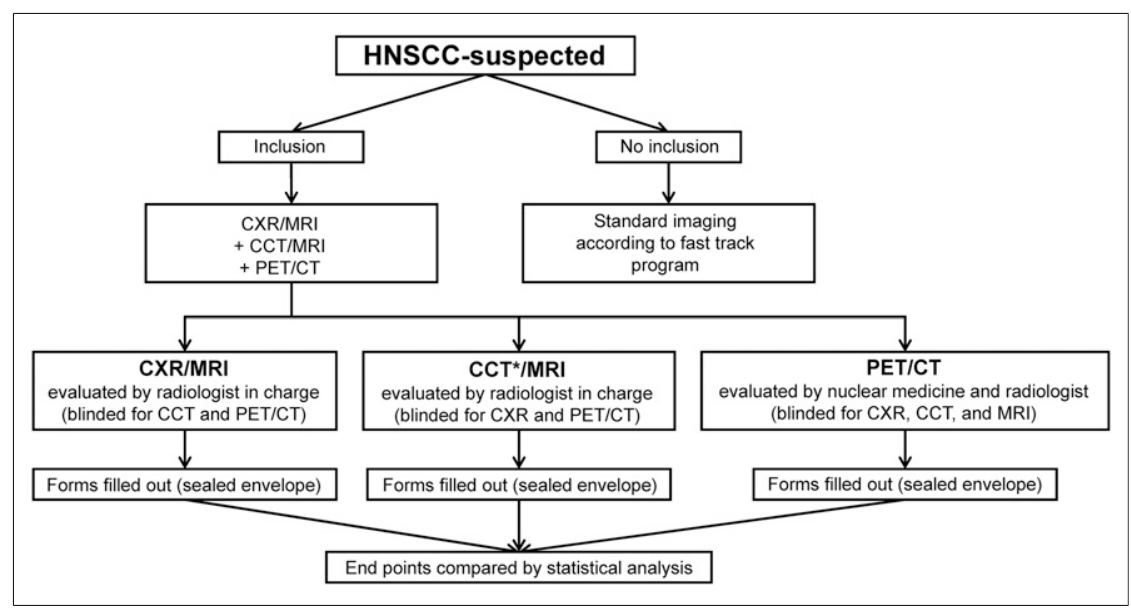

FIGURE 1. Diagram of patient inclusion and image interpretation. *Extracted from PET/CT. intravenous contrast medium (iopromide, $370 \mathrm{mg} / \mathrm{mL}$ [Ultravist; Bayer]) was acquired after the PET scan. The CT scan was obtained with continuous shallow breathing. Data were reconstructed with a standard filter into transaxial slices with a field of view of $50 \mathrm{~cm}$, a matrix size of $512 \times$ 512 (pixel size, $0.98 \mathrm{~mm}$ ), and a slice thickness of $3.75 \mathrm{~mm}$. The scan field of view was $70 \mathrm{~cm}$ for both PET and CT scans. The PET/CT data were analyzed on an Advantage Workstation (version 4.4 or 4.3; GE Healthcare) or on an AW Server (version 3.1 or 3.2; GE Healthcare).

MRI was performed on Achieva, Achieva dStream, or Ingenia 1.5-T hardware (Philips) using a 20-channel head-neck coil (dStream; Philips). The examination protocol was kept unchanged for the duration of the study and consisted of short-inversion-time inversion recovery, turbo spin echo T2-weighted, and turbo spin echo T1-weighted sequences with and without contrast enhancement, in the axial or coronal planes, with coverage from the skull base to the aortic arch using 5-mm slices. Diffusion-weighted imaging with spectral fat saturation, and apparent diffusion coefficient maps derived from b-values of 0 and $1,000 \mathrm{~mm}^{2} / \mathrm{s}$, were done in axial 6-mm slices. Images were read on a Centricity RA1000 PACS workstation (GE Healthcare). The intravenous contrast medium was gadoterate meglumine (Dotarem; Guerbet LLC), $279.3 \mu \mathrm{g} / \mathrm{mL}$ (body weight $<60 \mathrm{~kg}$ : $0.2 \mathrm{~mL} / \mathrm{kg}$; body weight $>60 \mathrm{~kg}$ and $<85 \mathrm{~kg}$ : $15 \mathrm{~mL} / \mathrm{kg}$; body weight $>85 \mathrm{~kg}: 20 \mathrm{~mL} / \mathrm{kg}$ ). The acquisition parameters for the MRI sequences are displayed in Supplemental Table 1 (supplemental materials are available at http://jnm.snmjournals.org).

Chest radiography was performed according to local standards. Anteroposterior and lateral projections with $130-145 \mathrm{kV}$ and automatic exposure control were acquired during full inspiration. FD-X hardware systems (Siemens Healthineers) were used, and studies were read using the Centricity RA1000 PACS workstation with dual 3-megapixel medical-grade monitors.

\section{Image Interpretation}

The applied imaging modalities were evaluated separately. The head and neck MRI scans and the chest radiographs were evaluated by 2 experienced head and neck radiologists, the chest CT scans were evaluated by 1 radiologist, and the PET/CT scans were evaluated by a team of 2 experienced radiologists and 2 nuclear physicians. Standard forms, including variables concerning the Union for International Cancer Control (UICC) stages (11), were completed by each diagnostic team during imaging evaluation. The same referral text was used for each of the evaluation sessions, and the teams were masked to one another.

Interpretation of primary tumors was based on anatomic pattern recognition, including morphologic changes, altered signal intensity, contrast enhancement, and changes in diffusivity, as well as metabolic information from ${ }^{18} \mathrm{~F}-\mathrm{FDG}$ avidity on PET. Malignant lesions were suspected when deemed more probable than benign lesions.

Characteristics considered for lymph nodes were enlargement, shape (round or not), hilum (fatty or not), necrosis, center (dense or not), topography of node distribution, and ${ }^{18}$ F-FDG avidity on PET.

For CT, lung lesions were labeled as distant metastasis if one or more nodules were present. Small subpleural nodules on CT, particularly when calcified, were not considered metastasis unless multiple nodules were present. The ${ }^{18} \mathrm{~F}$ FDG avidity of the nodules on PET/CT assisted in determining whether these lesions were malignant. Lung lesions in the field of view of the MRI scan were suspected of being metastasis if they had a nodular or specular configuration that was inconsistent with an infectious pattern. 
Likewise, on the chest radiograph, distant metastasis was suspected when opacification was not consistent with an infectious pattern.

Bone lesions on PET/CT were considered metastasis when ${ }^{18} \mathrm{~F}$ FDG-avid osteolytic (or osteosclerotic) lesions were present. Significantly increased ${ }^{18} \mathrm{~F}-\mathrm{FDG}$ uptake in the bone marrow, regardless of the presence of lytic or sclerotic changes, was also considered to be metastasis. Lesions close to the joints were rarely considered metastasis. With respect to MRI, focal signal changes on T2-weighted or short-inversion-time inversion recovery images accompanied by the presence of contrast enhancement were examined, but because of their variation, only lesions with a low T1-weighted signal were suspected of being metastasis. Osteolytic or osteosclerotic changes on chest radiography were suspected of being bone metastasis.

For PET/CT, liver metastasis and malignant pleural effusion were suspected only in lesions with significantly increased ${ }^{18} \mathrm{~F}-\mathrm{FDG}$ uptake in comparison with the surroundings. Muscle metastasis was suspected in patients with randomly distributed focal areas of increased ${ }^{18} \mathrm{~F}-\mathrm{FDG}$ uptake in the muscles as well as corresponding morphologic changes. Finally, longitudinal muscular ${ }^{18} \mathrm{~F}$-FDG uptake was considered to be physiologic.

\section{Sample Size Calculation}

The sample size estimation was based on the expectation of a $4 \%$ detection rate of distant metastasis by PET/CT (i.e., including patients with a low risk of distant metastasis, such as those with small glottis cancers), a $20 \%$ dropout, and detection by both CXR/MRI and PET/ CT in $2 \%$ of cases. Using the McNemar test at an $\alpha$ of 0.05 and a power of 0.90 , the estimated sample size was 366 patients. A predetermined interim analysis of 149 patients demonstrated a detection rate of $8 \%$ for PET/CT and only $1 \%$ for CXR/MRI, with a dropout rate of less than 5\%; thus, an adequate sample size was obtained.

\section{Outcomes}

The main outcome measure was the ability of cancer stages to discriminate survival, stratified by imaging modality. UICC stages were categorized to represent localized (stage I or II), locally advanced (stage III, IVA, or IVB), or metastatic (stage IVC) disease. Allcause mortality was used as the clinical outcome to assess the discriminative ability of HNSCC stage. Follow-up data on mortality were obtained by reviewing the patients' medical records for at least 6 mo (until August 31,2016 ) after termination of inclusion.

\section{Statistical Methods}

Continuous variables are presented as medians and ranges, and categoric variables are presented as counts and corresponding percentages. Overall comparisons of CXR/ MRI or CCT/MRI versus PET/CT for staging of HNSCC were conducted using the Pearson $\chi^{2}$ test, with patients stratified as having localized, locally advanced, or metastatic disease for each imaging modality. KaplanMeier analysis (21), including the log-rank test, and Cox proportional hazards regression with the Harrell concordance index (C-index) $(22,23)$ were used to compare the discriminative abilities of PET/CT versus CXR/MRI or CCT/MRI for stage-based survival. Finally, the ability of PET/CT to enhance accurate determination of mortality was tested with the net reclassification improvement (NRI) $(24,25)$. NRI quantifies the improvement in risk prediction offered by a new model compared with an old model by assessing the ability of the new model to appropriately reclassify subjects (i.e., into a higher or lower risk category). Thus, in the present study, the NRI, calculated on the basis of altered tumor staging, pertained only to survival rates and not to whether the actual cancer stage was in fact correctly determined.

The significance level was 5\%. All analyses were performed with Stata/IC 14 (StataCorp LP).

\section{RESULTS}

A patient-selection flowchart is presented in Figure 2. In total, 307 patients were included in the study, with basic clinical characteristics as outlined in Table 1. Supplemental Table 2 displays the location of metastasis in the 18 patients with histologically verified distant spread.

The distributions of localized (stages I or II), locally advanced (stages III, IVA, or IVB), and metastatic (stage IVC) disease are displayed in Table 2, stratified by imaging modality. CXR/MRI staged $45 \%$ of HNSCC cases as localized, $54 \%$ as locally advanced, and $1 \%$ as metastatic, whereas CCT/MRI staged $34 \%$ as localized, $40 \%$ as locally advanced, and $26 \%$ as metastatic. Finally, PET/CT staged $30 \%$ as localized, $62 \%$ as locally advanced, and $8 \%$ as metastatic. PET/CT caused changes in tumor stage, with a preponderance toward a more severe stage than with CXR/MRI and fewer metastatic cases than with CCT/MRI (both statistically significant at $P<0.001$ ).

\section{Hazard Risk}

The Kaplan-Meier plots in Figure 3 show the unadjusted survival rates for localized, locally advanced, and metastatic HNSCC as assessed by CXR/MRI, CCT/MRI, and PET/CT. When strictly considering stage-based differentiation of survival, CXR/MRI

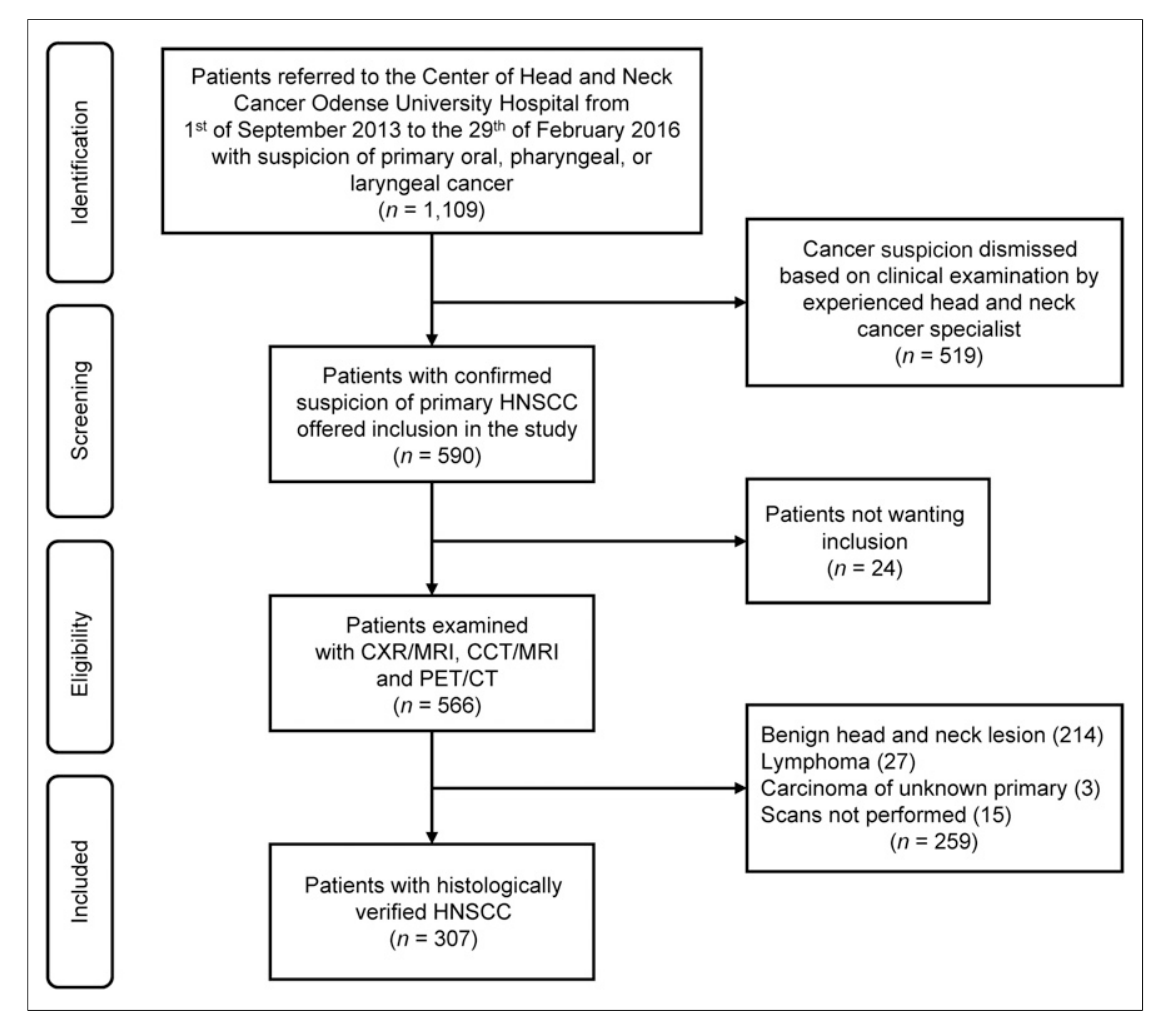

FIGURE 2. Flowchart of patient selection. 
TABLE 1

Baseline Clinical Characteristics

\begin{tabular}{|cc}
\hline \multicolumn{1}{c}{ Characteristic } & Data \\
\hline Age $(y)$ & 64 \\
\hline Median & $22-89$ \\
\hline Range & \\
\hline Sex $(n)$ & $227(74 \%)$ \\
\hline Male & $80(26 \%)$ \\
Female & \\
\hline Tumor site $(n)$ & $147(48 \%)$ \\
\hline Oral cavity & $103(34 \%)$ \\
Pharynx & $57(18 \%)$ \\
\hline Larynx & \\
\hline Verified distant metastasis* $(n)$ & $289(94 \%)$ \\
\hline M0 & $18(6 \%)$ \\
\hline M1 & \\
\hline p16 status $(n)$ & $242(79 \%)$ \\
\hline Negative & $65(21 \%)$ \\
\hline Positive & 461 \\
\hline Follow-up (d) & $21-1,092$ \\
\hline Median & \\
Range & \\
\hline
\end{tabular}

${ }^{*} 84 \%$ of cases were confirmed with follow-up imaging (within 6 $\mathrm{mo}), 8 \%$ with histology, and $8 \%$ with cytology. When only imaging was available as a validator, we applied RECIST for measurable lesions and progression. Only lesions fulfilling RECIST for progression were characterized as metastatic disease.

could not separate metastatic from localized $(P=0.17)$ or locally advanced HNSCC $(P=0.79)$, whereas CCT/MRI was unable to separate locally advanced from metastatic HNSCC $(P=0.94)$. Conversely, PET/CT significantly differentiated all groups in pairs; that is, when stages were determined by PET/CT, a significantly worse prognosis was found for locally advanced disease than for localized disease, for metastatic disease than for localized disease, and for metastatic disease than for locally advanced disease ( $P \leq 0.002$ for all comparisons). The adjusted hazard ratios confirmed these findings, with CCT/MRI further displaying a numerically higher risk of death in the locally advanced group than in the metastatic group (Table 3). When tumor staging was based on PET/CT, survival in the locally advanced and metastatic groups was significantly different from that in the localized group.

PET/CT had significantly better overall discriminative ability (C-index, 0.712) than CXR/MRI (C-index, 0.675; $P=0.04)$ or CCT/MRI (C-index, 0.657; $P=0.02$ ) for prediction of all-cause mortality.

\section{Reclassification}

The addition of PET/CT to either CXR/MRI or CCT/MRI was associated with a positive NRI, although this was significant only for the comparison with CXR/MRI (NRI, $18.4 \%$; 95\% confidence interval, $2.2 \%-34.6 \%$ ), not for CCT/MRI (NRI, 9.4\%; 95\% confidence interval, $-8.6 \%-27.4 \%$ ). PET/CT correctly upstaged
$35.5 \%$ of patients already staged by CXR/MRI (Supplemental Table 3) and correctly downstaged $24.5 \%$ of patients already staged by CCT/MRI (Supplemental Table 4). As previously mentioned, NRI was calculated on the basis of altered tumor stage and pertained only to survival rates; that is, "correctness" was unrelated to whether the actual cancer stage was correctly determined.

\section{DISCUSSION}

Our results demonstrate that a PET/CT-based imaging strategy was associated with significant changes in assigned tumor stage and that PET/CT was associated with significantly improved discrimination between tumor stages compared with CXR/MRIor CCT/MRI-based strategies, when considering survival among HNSCC patients.

Specifically, CXR/MRI had low sensitivity for metastatic disease; this combination was not able to identify patients with a poor prognosis. At the other end, CCT/MRI was unable to separate locally advanced disease from metastatic disease in such a way that their resulting mortality rates differed; that is, the specificity was low. PET/CT correctly reclassified a large number of patients, stratified by either of the other imaging modalities, and thus displayed useful prognostic properties.

Previous studies have focused on diagnostic accuracy mainly in terms of TNM classification and less on UICC staging or, importantly, on their significance with respect to a clinically important hard endpoint $(7,14,26-30)$. For diagnostic purposes, previous reports have generally shown that the addition of PET/ CT to CCT/MRI resulted in higher staging accuracy, particularly regarding the specificity of $\mathrm{N}$ and $\mathrm{M}$ classification. The same authors have speculated that PET/CT might improve prognostic accuracy, but although they found PET/CT imaging to be effective for diagnostic TNM classification of HNSCC, the resulting clinical implications in terms of survival were not examined.

Ryu et al. (31) examined the prognostic implications of adding PET/CT (after histopathology) to standard clinical imaging - that is, including CT or MRI of the head and neck and CT of the chest-in 248 patients with HNSCC. The investigators showed

TABLE 2

Distribution According to UICC Stage and Imaging Modality

\begin{tabular}{|cccc}
\hline \multicolumn{1}{c}{ Stage } & CXR/MRI $^{*}$ & CCT $^{\dagger} / M^{*} I^{*}$ & PET/CT \\
\hline Localized & $137(45)$ & $103(34)$ & $94(30)$ \\
\hline Stage 0 & $29(9)$ & $19(6)$ & $13(4)$ \\
Stage I & $75(25)$ & $58(19)$ & $62(20)$ \\
Stage II & $33(11)$ & $26(9)$ & $19(6)$ \\
Locally advanced & $167(54)$ & $123(40)$ & $190(62)$ \\
\hline Stage III & $53(17)$ & $42(14)$ & $38(12)$ \\
Stage IVA & $110(36)$ & $77(25)$ & $149(49)$ \\
Stage IVB & $4(1)$ & $4(1)$ & $3(1)$ \\
\hline Metastatic (stage IVC) & $3(1)$ & $81(26)$ & $23(8)$ \\
\hline
\end{tabular}

*Same MRI scan was used for staging with chest radiography and chest $\mathrm{CT}$.

${ }^{\dagger}$ Extracted from PET/CT.

Data are $n$ followed by percentage in parentheses. 


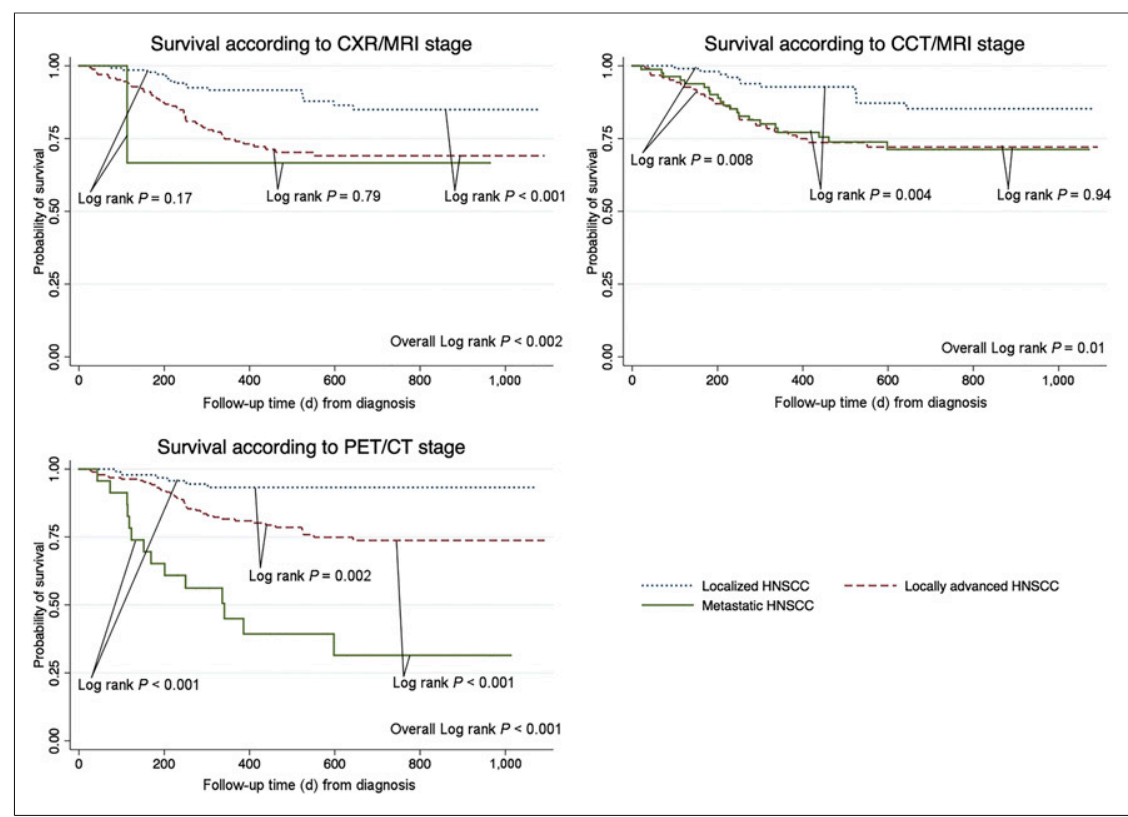

FIGURE 3. Unadjusted Kaplan-Meier estimates of survival in HNSCC.

that patients whose disease was upstaged by PET/CT, compared with a conventional imaging workup, had significantly worse progression-free and overall survival than patients whose stage was not altered. However, no direct comparison with standard imaging was performed, and the discriminative ability among stages was not assessed.

Thus, our study directly assessed the clinical value of upfront PET/CT, compared with CXR/MRI and CCT/MRI, in the diagnostic workup of patients with HNSCC. Further methodologic variations in previous studies included the use of different imaging technologies, that is, PET alone versus PET/CT, masking, and reference standards.

TABLE 3

Hazard Ratios and 95\% Confidence Intervals from Age- and Sex-Adjusted Cox Regression Models for Cancer Staging According to Imaging Modality

\begin{tabular}{lccc}
\hline \multicolumn{1}{c}{ Modality } & Hazard ratio & $\begin{array}{c}\text { Confidence } \\
\text { interval }\end{array}$ & $P$ \\
\hline CXR/MRI $^{*}$ & & & \\
\hline $\begin{array}{l}\text { Locally advanced }^{\text {Metastatic }} \\
\quad\end{array}$ & 2.8 & $1.6-5.0$ & $<0.001$ \\
CCT'/MRI $^{*}$ & 4.9 & $0.6-37.6$ & 0.13 \\
\hline $\begin{array}{l}\text { Locally advanced } \\
\text { Metastatic }\end{array}$ & 2.7 & $1.4-5.5$ & 0.004 \\
\hline PET/CT & 2.5 & $1.2-5.2$ & 0.01 \\
\hline $\begin{array}{l}\text { Locally advanced } \\
\text { Metastatic }\end{array}$ & 3.5 & $1.5-8.2$ & 0.004 \\
\end{tabular}

*Same MRI scan was used for staging with chest radiography and chest CT.

${ }^{\dagger}$ Extracted from PET/CT.

Localized HNSCC serves as baseline for all comparisons.
The current study extends previous findings by confirming the suggested clinical implications of $\mathrm{PET} / \mathrm{CT}$ in a prospective study design. Despite a relatively short follow-up, PET/CT was associated with improved overall discriminative ability, clinically meaningful stage separation, and a theoretic added value in clinical scenarios where conventional images are obtained before PET/CT. The results also provide a rationale for avoiding a sequential imaging strategy and performing PET/CT alone instead. Hypothetically, these properties of PET/CT not only should provide a more accurate estimation of prognosis but also should increase the likelihood of a more optimized treatment strategy.

This study directly compared the clinical value of upfront PET/CT with the traditional imaging techniques recommended by contemporary national clinical practice guidelines, including those from the National Comprehensive Cancer Network (10), and various contemporary European guidelines $(8,9,32)$. At present, the European guidelines recommend PET/CT only for evaluating patients with malignant cervical adenopathy from an unknown primary tumor, whereas the U.S. guidelines consider PET/CT for clinical stage 3-4 disease. However, the results indicate that upfront PET/CT facilitates a more clinically meaningful tumor staging of HNSCC patients in terms of prognosis and may be preferable in the diagnostic work-up of patients with HNSCC. Introduction of upfront PET/CT might reduce the number of imaging procedures required, improve staging in terms of survival, and thus establish the best basis for individual treatment planning.

Our findings can be put into further perspective by highlighting the fact that studies on patients with lung cancer have shown similar patterns of stage migration for PET/CT, with improved prognostic discrimination using Kaplan-Meier analyses $(33,34)$. Furthermore, significant prognostic implications for PET/CT in HNSCC patients have already been demonstrated in the setting of surveillance for recurrent disease after completion of chemoradiotherapy $(35,36)$.

The strengths of our study include the prospective design, inclusion of consecutive patients over a $2.5-y$ period, use of each patient as his or her own control, upfront imaging with all modalities on the same day and with up-to-date technology, and expert assessment with masking of the results of the other modalities. The strengths and validity of paired diagnostic studies versus randomized (controlled) studies have been well described (37-40). The paired data design eliminates the risk of confounding and the need for stratified analysis, with further advantages being the dependence of the sample size on the agreement rate between the modalities, the ability to meet the multiple aims of diagnostic accuracy studies, and the possibility of early unmasking of results at the individual level. For these reasons, we deliberately chose a paired data design rather than a randomized one.

A few limitations deserve comment. The drawback of upfront imaging is that a considerable proportion of patients undergoes 
imaging without having malignant disease. Therefore, a substantial clinical suspicion of cancer must be present to minimize radiation exposure and costs and to ensure relevant indications for this diagnostic strategy. The diagnostic yield of whole-body PET/ $\mathrm{CT}$ in patients with clinically localized disease is also uncertain. Routine PET/CT in all patients with newly diagnosed HNSCC should therefore be carefully considered. Additionally, a high number of negative PET/CT results (outside the neck) and a possible increase in the detection of false-positive findings outside the neck may occur. Furthermore, in the case of surgery, highly detailed images in multiple planes are needed, and these are best provided by head and neck MRI or CT with intravenous contrast medium. Thus, PET alone (without fully diagnostic CT) may be insufficient for patients who are potential candidates for surgery (12).

This study dealt with the ability of each imaging modality to provide a clinically meaningful separation of cancer stages; that is, when resultant apparent stages were distinct in terms of the survival provided, better statistical measures were obtained. Since no comparison with a histopathologic reference standard (the gold standard) was made, the accuracy of the individual imaging modalities for "correct" staging per se could not be assessed. The calculated NRI, in accordance with its definition and purpose, was related strictly to survival as well. Unfortunately, no details on treatment strategy were available. However, the actual treatment strategy for the patients included in this study was based on a comprehensive assessment of all available imaging studies, including PET/CT. Therefore, the resulting predictive value for mortality may have been overestimated, provided the main cause of death was cancer-related.

An additional limitation of our study was that the CT scan was obtained with continuous shallow breathing. Standard chest CT is performed at breath-hold, which is more sensitive, particularly for basal lung nodules. Comparing PET/CT, which is a whole-body scan, with regional radiologic imaging modalities may also seem unfair. However, the aim of this study was to compare the clinical imaging strategies used today in most head and neck cancer centers.

Furthermore, the study was performed at a single institution, possibly restricting the generalizability of the results. Although patients suspected of having oral cavity cancer were referred from the entire Region of Southern Denmark, patients with suspected laryngeal or pharyngeal cancer were referred from the smaller region of Funen, with the result that oral cavity cancer was oversampled. Lastly, disease-specific mortality would have been a clinical endpoint preferable to all-cause mortality.

\section{CONCLUSION}

Tumor stages determined by PET/CT were associated with more distinct prognostic properties in terms of survival than those determined by standard imaging modalities.

\section{DISCLOSURE}

This study was financially supported by the University of Southern Denmark, the Danish Cancer Society, and the Region of Southern Denmark. Doctoral research grants were given by the University of Southern Denmark, the Danish Cancer Society, and the Region of Southern Denmark. No other potential conflict of interest relevant to this article was reported.

\section{REFERENCES}

1. Lassen P. The role of human papillomavirus in head and neck cancer and the impact on radiotherapy outcome. Radiother Oncol. 2010;95:371-380.

2. Benson E, Li R, Eisele D, Fakhry C. The clinical impact of HPV tumor status upon head and neck squamous cell carcinomas. Oral Oncol. 2014;50:565-574.

3. VanderWalde NA, Salloum RG, Liu TL, et al. Positron emission tomography and stage migration in head and neck cancer. JAMA Otolaryngol Head Neck Surg. 2014;140:654-661.

4. Lonneux M, Hamoir M, Reychler H, et al. Positron emission tomography with $\left[{ }^{18} \mathrm{~F}\right]$ fluorodeoxyglucose improves staging and patient management in patients with head and neck squamous cell carcinoma: a multicenter prospective study. J Clin Oncol. 2010;28:1190-1195.

5. Paidpally V, Tahari AK, Lam S, et al. Addition of ${ }^{18} \mathrm{~F}-\mathrm{FDG}$ PET/CT to clinical assessment predicts overall survival in HNSCC: a retrospective analysis with follow-up for 12 years. J Nucl Med. 2013;54:2039-2045.

6. Connell CA, Corry J, Milner AD, et al. Clinical impact of, and prognostic stratification by, F-18 FDG PET/CT in head and neck mucosal squamous cell carcinoma. Head Neck. 2007;29:986-995.

7. Rohde M, Dyrvig AK, Johansen J, et al. ${ }^{18}$ F-fluoro-deoxy-glucose-positron emission tomography/computed tomography in diagnosis of head and neck squamous cell carcinoma: a systematic review and meta-analysis. Eur J Cancer. 2014;50: 2271-2279.

8. Grégoire V, Lefebvre JL, Licitra L, Felip E. Squamous cell carcinoma of the head and neck: EHNS-ESMO-ESTRO clinical practice guidelines for diagnosis, treatment and follow-up. Ann Oncol. 2010;21:v184-v186.

9. Pakkeforløb for hoved- og halskrft. Sundhedsstyrelsen website. https://www.sst. $\mathrm{dk} / \mathrm{da} /$ sygdom-og-behandling/kraeft/pakkeforloeb/ /media/

94B4013E3D2D445DAC2494EB1CA194CE.ashx. Published January 29, 2016. Accessed January 31, 2018.

10. Pfister DG, Spencer S, Adelstein D, et al. Head and Neck Cancers: Version 1.2016. Fort Washington, PA: National Comprehensive Cancer Network; 2017.

11. Leslie H. Sobin MKG, Christian Wittekind, ed. TNM Classification of Malignant Tumours. 7th ed. Geneva, Switzerland: Union for International Cancer Control (UICC); 2009.

12. Yoo J, Henderson S, Walker-Dilks C. Evidence-based guideline recommendations on the use of positron emission tomography imaging in head and neck cancer. Clin Oncol (R Coll Radiol). 2013;25:e33-e66.

13. Gordin A, Daitzchman M, Doweck I, et al. Fluorodeoxyglucose-positron emission tomography/computed tomography imaging in patients with carcinoma of the larynx: diagnostic accuracy and impact on clinical management. Laryngoscope. 2006;116:273-278.

14. Xu GZ, Guan DJ, He ZY. ${ }^{18}$ FDG-PET/CT for detecting distant metastases and second primary cancers in patients with head and neck cancer: a meta-analysis. Oral Oncol. 2011;47:560-565.

15. Kim SY, Roh JL, Yeo NK, et al. Combined ${ }^{18} \mathrm{~F}$-fluorodeoxyglucose-positron emission tomography and computed tomography as a primary screening method for detecting second primary cancers and distant metastases in patients with head and neck cancer. Ann Oncol. 2007;18:1698-1703.

16. Agra IMG, Ferlito A, Takes RP, et al. Diagnosis and treatment of recurrent laryngeal cancer following initial nonsurgical therapy. Head Neck. 2012;34: 727-735.

17. Johansen J, Petersen H, Godballe C, Loft A, Grau C. FDG-PET/CT for detection of the unknown primary head and neck tumor. $Q J$ Nucl Med Mol Imaging. 2011;55:500-508.

18. Vandenbroucke JP, von Elm E, Altman DG, et al. Strengthening the Reporting of Observational Studies in Epidemiology (STROBE): explanation and elaboration. Int J Surg. 2014;12:1500-1524.

19. Rohde M, Nielsen AL, Johansen J, et al. Head-to-head comparison of chest $\mathrm{x}$-ray/head and neck MRI, chest CT/head and neck MRI, and ${ }^{18} \mathrm{~F}-\mathrm{FDG}$ PET/CT for detection of distant metastases and synchronous cancer in oral, pharyngeal, and laryngeal cancer. J Nucl Med. 2017;58:1919-1924.

20. Grau C, Toustrup K, Christensen A, et al. Fast track for head and neck cancer patients: the story of the Danish national strategy for accelerated diagnosis and treatment of cancer [abstract]. Radiother Oncol. 2011;99(suppl 1):S209.

21. Kaplan ELMP. Nonparametric estimation from incomplete observations. J Am Statist Assn. 1958;53:457-481.

22. Harrell FE Jr, Califf RM, Pryor DB, Lee KL, Rosati RA. Evaluating the yield of medical tests. JAMA. 1982;247:2543-2546.

23. Harrell FE Jr, Lee KL, Mark DB. Multivariable prognostic models: issues in developing models, evaluating assumptions and adequacy, and measuring and reducing errors. Stat Med. 1996;15:361-387.

24. Greenland P. Comments on 'Evaluating the added predictive ability of a new marker: from area under the ROC curve to reclassification and beyond' 
by M. J. Pencina, R. B. D'Agostino Sr, R. B. D'Agostino Jr, R. S. Vasan, Statistics in Medicine (DOI: 10.1002/sim.2929). Stat Med. 2008;27:188-190.

25. Pencina MJ, D'Agostino RB Sr, D'Agostino RB Jr, Vasan RS. Evaluating the added predictive ability of a new marker: from area under the ROC curve to reclassification and beyond. Stat Med. 2008;27:157-172.

26. Roh JL, Yeo NK, Kim JS, et al. Utility of $2-\left[{ }^{18} \mathrm{~F}\right]$ fluoro-2-deoxy-D-glucose positron emission tomography and positron emission tomography/computed tomography imaging in the preoperative staging of head and neck squamous cell carcinoma. Oral Oncol. 2007;43:887-893.

27. Abramyuk A, Appold S, Zophel K, Baumann M, Abolmaali N. Modification of staging and treatment of head and neck cancer by FDG-PET/CT prior to radiotherapy. Strahlenther Onkol. 2013;189:197-201.

28. Scott AM, Gunawardana DH, Bartholomeusz D, Ramshaw JE, Lin P. PET changes management and improves prognostic stratification in patients with head and neck cancer: results of a multicenter prospective study. J Nucl Med. 2008; 49:1593-1600

29. Cacicedo J, Fernandez I, Del Hoyo O, et al. Should PET/CT be implemented in the routine imaging work-up of locally advanced head and neck squamous cell carcinoma? A prospective analysis. Eur J Nucl Med Mol Imaging. 2015;42:1378-1389.

30. Nair S, Mohan S, Nilakantan A, Gupta A, Malik A, Gupta A. Impact of ${ }^{18}$ f-fluorodeoxyglucose positron emission tomography/computed tomography scan on initial evaluation of head and neck squamous cell carcinoma: our experience at a tertiary care center in India. World J Nucl Med. 2015;14:19-24.

31. Ryu IS, Roh JL, Kim JS, et al. Impact of ${ }^{18} \mathrm{~F}$-FDG PET/CT staging on management and prognostic stratification in head and neck squamous cell carcinoma: a prospective observational study. Eur J Cancer. 2016;63:88-96.
32. Lewis-Jones H, Colley S, Gibson D. Imaging in head and neck cancer: United Kingdom national multidisciplinary guidelines. J Laryngol Otol. 2016;130:S28S31.

33. Geiger GA, Kim MB, Xanthopoulos EP, et al. Stage migration in planning PET/ $\mathrm{CT}$ scans in patients due to receive radiotherapy for non-small-cell lung cancer. Clin Lung Cancer. 2014;15:79-85.

34. Tönnies S, Bauer TT, Misch D, et al. Staging with ${ }^{18}$ F-FDG-PET/CT influences stage-specific survival in advanced non-small cell lung cancer (NSCLC) [in German]. Pneumologie. 2012;66:212-217.

35. Kim JW, Roh JL, Kim JS, et al. ${ }^{18} \mathrm{~F}-\mathrm{FDG}$ PET/CT surveillance at 3-6 and 12 months for detection of recurrence and second primary cancer in patients with head and neck squamous cell carcinoma. Br J Cancer. 2013;109:2973-2979.

36. Kim SA, Roh JL, Kim JS, et al. ${ }^{18}$ F-FDG PET/CT surveillance for the detection of recurrence in patients with head and neck cancer. Eur J Cancer. 2017;72: $62-70$.

37. Gerke O, Hoilund-Carlsen PF, Poulsen MH, Vach W. Interim analyses in diagnostic versus treatment studies: differences and similarities. Am J Nucl Med Mol Imaging. 2012;2:344-352.

38. Siepe B, Hoilund-Carlsen PF, Gerke O, Weber WA, Motschall E, Vach W. The move from accuracy studies to randomized trials in PET: current status and future directions. J Nucl Med. 2014;55:1228-1234.

39. Vach W, Hoilund-Carlsen PF, Gerke O, Weber WA. Generating evidence for clinical benefit of PET/CT in diagnosing cancer patients. J Nucl Med. 2011;52 (suppl 2):77S-85S.

40. Gerke O, Hoilund-Carlsen PF, Vach W. Analyzing paired diagnostic studies by estimating the expected benefit. Biom J. 2015;57:395-409. 